\title{
"Live - Die - Repeat" The Time Loop as a Narrative and a Game Mechanic
}

\section{Linda Lahdenperä}

University of Jyväskylä, Finland

DOI: https://doi.org/10.7358/ijtl-2018-006-lahd_ｌilian.lahdenpera@gmail.com

ABSTRACT - Characters in time loop narratives, like players of video games, repeat specific sequence of events with varying outcomes to reach a goal. In this article time loop is considered as a phenomenon that can either be experienced by characters inside the diegesis, by players on the level of game mechanics or by game characters and players both. My study has three aims: to use the time loop as an example on how films remediate video games, to conceptualize the time loop by applying the possible worlds model, and analyze the experience of parallel, mutually exclusive events. I find that time loop films resemble video games in their levellike and goal-oriented structure, as well as in their portrayal of death and in the lack of agency of the supporting characters. When conceptualizing the levels of time loops, I learn that both multilinear and linear games can be loops for players, even though the looping is not part of the diegesis. Also, the replaying of a game with a time loop narrative is only a loop for the player since game characters can only be aware of the looping inside a playthrough. However, replaying a game can weaken the narrative experience so some players choose not to replay and some game designers encourage it. Incorporating the ambiguity of mutually exclusive events to the diegesis might solve the issue.

“'Xena, you're saying that I forgot everything that happened yesterday?'

'You didn't just forget; it never happened to you.'

'Well, if it never happened, then what's to forget?'

'Today.'

'But today hasn't happened yet.'

'Well, it happened to me.'

'Yesterday.'

'No, the other today.'

'so you're saying that today is actually yesterday for you - but, for us, today is today, because we can't remember that yesterday was today, right?'

'Right.'” 
The dialogue above is an excerpt from one of the most popular episodes of television series Xena the Warrior Princess $(1995$ - 2001). In the episode fittingly titled "Been There Done That" (season 3, episode 2), Xena keeps living the same day over and over again. If that would not be bad enough she is the only one aware of the repetition and, to make matters worse, a blood bath will break out every time unless Xena figures out how to prevent it. She has one day to correct things before the same day begins yet again.

In popular culture Xena's predicament is known as time loop and Xena is not the only one caught in it. The trope can be found in novels, short stories, films, video games and television series. The most famous time loop narratives are probably the films Groundhog Day (1993) and Lola Rennt (Run Lola Run) (1998). The trope's popularity has continued into the 2000s and 2010s with blockbuster films such as Source Code (2008) and Edge of Tomorrow (2014), young adult fiction such as novel (2010) and film (2017) Before I Fall by Lauren Oliver and video game Life is Strange (Dontnod Entertainment 2015), to name but a few.

In recent studies Christoph Bode and Rainer Dietrich (2013), Jasmina Kallay (2013), Sabine Schenk (2013) and Victor Navarro-Remesal and Shaila García-Catalán (2015) among others have paid attention to the game-like nature of time loop narratives. A player of a video game, just like a character in a time loop narrative, often replays scenes or levels. If the player's avatar dies, time rewinds back and the avatar respawns; ready to try (and die) again until a goal has been reached. Time loop narratives, then, can be seen to imitate the replay quality of video games.

Jay David Bolter and Richard Grusin ([1999] 2000, 45) have coined the term remediation to refer to "the representation of one medium in another". Irina Rajewsky $(2005,52)$ also uses remediation to describe a situation where one media imitates another. This is also where the connection between time loop narratives and transmedia can be found. The classic definition of transmedia by Henry Jenkins (2007) goes: "Transmedia storytelling represents a process where integral elements of a fiction get dispersed systematically across multiple delivery channels for the purpose of creating a unified and coordinated entertainment experience. Ideally, each medium makes its own unique contribution to the unfolding of the story." Lisbeth Klastrup and Susana Tosca $(2014,296)$ define transmedial worlds as "abstract content systems from which a repertoire of fictional stories can be actualized or derived across a variety of media forms". Each medium has 
its own way of telling a story and these medium specific characteristics can influence each other. Furthermore, transmedia stories are often characterized by their playfulness (see Harvey 2015, 16) which is a quality also typical to time loop narratives. Even though they are not transmedia per se, time loop narratives can be seen belonging to the wider phenomenon of intermedial relations.

Juxtaposing a player of a video game with a character of a time loop narrative is problematic, however, because they exist on different ontological levels. The looping for the player occurs on the level of the game mechanics whereas for the game character it occurs inside the diegesis. From this it follows that a player can experience a time loop even when the game character cannot. For example playing Super Mario Bros (Nintendo 1985) might feel like a time loop for the player, although it is not a time loop story. To analyze these different levels of time loops and their implications for the player and game character is important in order to distinguish between story and mechanics. The analysis also provides tools to conceptualize a player's experience of the interaction between mechanics and story, which is of significance when designing a video game that can be replayed.

In this article I will, then, develop on the earlier research by considering time loop as both diegetic (taking place inside the fiction) and extradiegetic (taking place outside of fiction) experience. My article has three aims. Firstly, I aim to conceptualize the time loop from the point of view of the fictional character (either in a story or a game) and player and analyze different possibilities for time loops: as a narrative in a non-interactive media ${ }^{1}$, as a narrative in a video game and as game mechanics. Secondly, I will look at what video game characteristics can be identified from time loop films in order to illustrate remediation. Thirdly, I will analyze the paradoxical experience typical to time loops: when something both happens and does not happen and its effects on the player and fictional character.

Following Bode and Dietrich (2013) and Schenck (2013) I position time loops into the framework of future narratives. They are narratives that present the future as a possibility space where different mutually exclusive continuations can be explored. As an analytical method to conceptualize and compare different time loops I employ the possible worlds theory and the concept of fictional ontology as presented by Marie-Laure Ryan (1991;

${ }^{1}$ With non-interactive media I mean that the reader/viewer cannot influence what happens in the narrative.

International Journal of Transmedia Literacy - 4 - December 2018

http: //www.ledonline.it/transmedialiteracy - Online ISSN 2465-2261 - Print ISSN 2465-227X 
2006) and Raine Koskimaa (2000). Possible worlds theory assumes that a story is a universe consisting of multiple worlds and fictional ontology refers to the hierarchy of these worlds. Fictional ontology is closely related to another key concept of the article, which is the hierarchical structure of narration. In its most basic form it is about dividing the narrative text into story and discourse (Genette 1980; Chatman 1978), or in the case of video games into story and mechanics (or, following Mäyrä (2008, 17-18) into core and shell).

\section{TIME LOOP AS A FUTURE NARRATIVE}

Future narratives are narratives that stage the future as it appears to us in real life: open and undecided with multiple possibilities. However, unlike in real life, where we have to contend with exploring only one and imagining the others, in these narratives several possibilities are explored. Future narratives achieve this sense of openness by presenting at least one situation in such a way that it allows for more than one possible continuation. This is called a nodal situation (Bode and Dietrich 2013, 1).

There are two types of future narratives: the forking paths and time loop. They resemble each other and are sometimes confused with each other (see Bordwell 2002; Parshall 2012) but they differ in who focalizes the different possibilities explored. With focalization I mean from which character's literal and figurative point of view the events in the story are seen or experienced (Chatman 1978, 151-152; Bal 1997, 42-143).

In the time loop the events are focalized through one and the same character. That is, at least one character is aware of the repetition. A character will live through a specific period of time, and after that time period has ended (or the character has died), she will go back and repeat the same period of time and use what she learnt from previous rounds to change the outcome. In a forking paths narrative, on the other hand, a character enters a nodal situation but when that happens, the character splits into multiple versions of herself and each of these versions focalizes only one of the possible trajectories. These various versions of the same character do not get the chance to learn and select the best possible outcome because they are not aware of the repetition. To be precise, in forking paths the repetition is an artifice of the narrative medium, where events taking place simultane- 
ously are presented consequentially which creates a sense of looping ${ }^{2}$. Examples of forking paths narratives are films Przypadek (Blind Chance) (1981) and Sliding Doors (1998).

Both time loops and forking paths explore the various possible outcomes of a character's actions and choices. But where forking paths narratives concentrate on the (often long-term) consequences of actions and typically emphasize the role of coincidence, the focus of time loops is on learning from the (often short-term) consequences and eliminating coincidences. This is accomplished by repeatedly visiting and taking control of the same period of time.

There are two aspects of future narratives that are particularly important for the present article. They are agency and playfulness, which I will be discussing next in this order. According to Bode and Dietrich (2013, 7, 43) the one who has agency, in other words, the one making decisions and affecting which possibilities are actualized is called an agent. The agent can either be a character inside the narrative or a reader/player/viewer outside the narrative, depending on the media format and the level of interactivity of the narrative. Examples of future narratives with an outside agent include hypertexts, alternate reality games, Choose Your Own Adventure books and video games. In these interactive future narratives the reader/ player/viewer affects the narrative directly or through an avatar. Examples of future narratives with an agent inside the narrative include all films, television series and novels with multiple possible futures but where the viewer/reader cannot affect which options are actualized.

What is interesting here is that Bode and Dietrich make no distinction based on the ontological position of the agent. An agent is an agent whether she exists inside the story or outside of it, and a narrative is a future narrative if it offers multiple continuations regardless of its media format and where the agent is situated. From this it follows that Bode and Dietrich do not differentiate between whether the multiple options exist on the level of the story, on the level of the discourse (or mechanics in case of video games) or on both levels. I, however, postulate that the ontological and narrative levels do affect on how the narrative is experienced and how it appears, especially when it comes to video games. This is why in the following sections I will look at this matter more closely. But before moving on to that, I want to discuss the other important aspect of this article, which is playfulness.

${ }^{2}$ In films a split screen is sometimes employed to represent two parallel realities. 
To present the future as various possibilities to be chosen from has certain playfulness to it, and Schenk $(2013,135,140)$ calls future narratives in films, television and novels as metaphorical games. They have more in common with games than traditional storytelling. The structure of a forking paths narrative corresponds to the branching structure typical to many video games. The structure of a time loop narrative, as stated in the introduction, bares a strong resemblance to video game retry mechanics. The protagonists of future narratives, then, do different runs through the narrative architecture, like avatars in video games. A time loop film can be considered to be a completed and edited playthrough, akin to a Let's Play video. Here we loop back to discussion about agents in future narratives and their ontological positions. Since time loop narratives are narratives that resemble video games and both players and characters can act as agents in them, my aim is next to analyze the experiences and ontological positions of a player and character in time loop narratives and video game mechanics.

\section{THE ONTOLOGICAL HIERARCHY IN THE TIME LOOP}

Time loops occur on different narrative and ontological levels. In the Figure 1 the levels of the narrative are divided in two. There is first the extradiegetic level where the reader, viewer and the player exist, depending on the medium of the narrative. For the sake of simplicity, there is only the agent and observer in the figure. The difference is that the agent is the one both influencing and observing the events in the story whereas observer only observes the story unfolding. Depending on the medium of the narrative the agent can be a player or a reader of an interactive book or someone watching an interactive film. The observer, on the other hand, can be a reader or viewer of a non-interactive narrative or someone observing while someone else acts as the extradiegetic agent. (Fig. 1)

Inside the box there is the level of the story where the characters exist. There is a hierarchy between the characters depending on do they experience the looping or not. If there is an intradiegetic agent in the narrative, a character aware of and influencing the loop, then she is the highest in the hierarchy. Underneath the agent there are the characters who are not aware of the loop and then not influencing it and learning from it. For the sake of 
simplicity, however, in the figure there is now only the looping character and her counterparts in possible worlds. The thought behind this is that every time the specific period of time is repeated, it is repeated in a parallel world and the consciousness of the looping character merges with her counterpart in the parallel world (in the next section the theory of possible parallel worlds and the travel between them is discussed in more depth). If, however, there is no consciousness aware of the looping inside the story, then there are only several versions of the same character existing in parallel worlds. (Fig. 1)

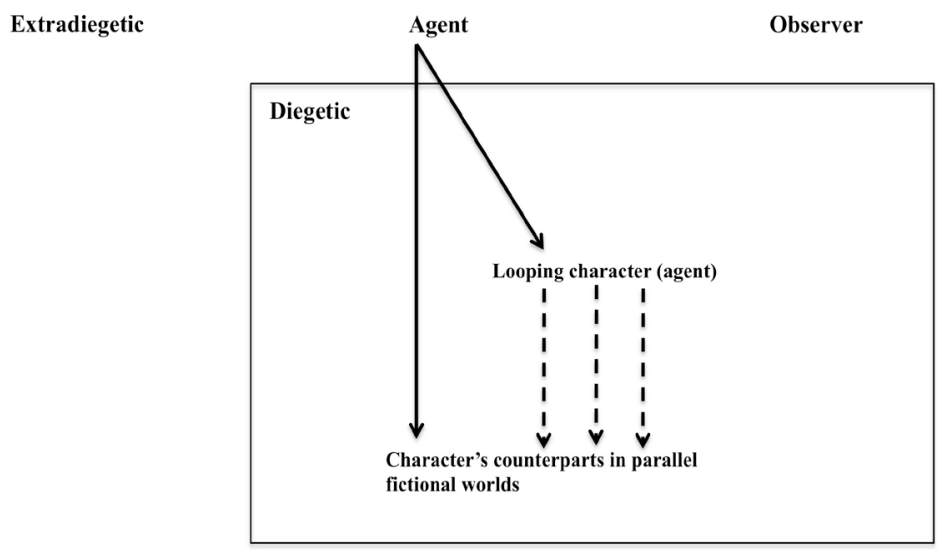

Figure 1. Ontological and narrative levels

Now let us look at the extradiegetic and diegetic levels at the same time and see what combinations there are for time loops. The first option is that there is no outside agent, only the observer, and the agent is located inside the narrative. In this case it is the looping character who acts as the agent merging with her counterparts in possible parallel worlds. The looping character is in a somewhat similar position as the player of a video game but she is (usually) her own avatar. This is a time loop story in a non-interactive narrative such as a film, novel or television series.

The second option is that the agent exists outside of the fictional world. In this case the agent influences what happens in the story either directly or in a typical case via an avatar inside the story. This is symbolized 
by the arrow on the left. The looping only occurs outside of the story (on the level of the discourse or mechanics) for the agent since the avatar is not aware of the looping and is only a vessel for the extradiegetic agent. This is then not a time loop narrative but the loop is nevertheless experienced by the extradiegetic agent. This agent is most often a player of a video game ${ }^{3}$.

The third and final option is that there is an agent both on the extradiegetic and diegetic level. This is symbolized by the arrow on the right. The extradiegetic agent controls a character who is aware of the looping. This character in turn merges with her counterparts in possible parallel worlds. In this option the outside agent has more control than the inside agent, however the inside agent typically is not just a vessel but, depending on the script, has some autonomous agency too. This is what happens when a video game has a time loop narrative.

Now that we have analyzed the levels and possibilities to experience the time loop let us move on to discuss how the looping can occur. In the following section I will use the possible worlds theory in conceptualizing the time loop and show different examples of time loops depending on the ontological position of the agent.

\section{CONCEPTUALIZING THE TIME LOOP}

Time loops are spaces where one thing can happen in more than one way. An agent can for example both get hit by a car and not get hit by the car (directly, if the agent is inside the narrative, or indirectly via an avatar, if the agent is outside the narrative). This state of affairs seems at first counterintuitive since in our world things only happen one way. However, a means to conceptualize this is to suggest that each of the versions of the same events happen in a different world.

Possible worlds theory in narratology is adapted from modal logic, which is a branch of formal semantics in analytic philosophy. Modal logic is the study of truth conditions of expressions, which state something is necessary or possible. Modal logic uses the metaphor of world to describe the

${ }^{3}$ If there is no extradiegetic agent and no character inside the story aware of the looping, only the various counterparts of a character in parallel worlds, then it is the case of a forking paths narrative.

International Journal of Transmedia Literacy - 4 - December 2018

http: //www.ledonline.it/transmedialiteracy - Online ISSN 2465-2261 - Print ISSN 2465-227X 
semantic domain projected by the text and the concept of modality to describe the various ways the objects, states and events in the text can exist (Ryan 1991, 3; Ryan 2006, 644) Because of this, possible worlds theory is useful when analyzing narratives with conflicting and mutually exclusive events (Koskimaa 2000, 66-73).

The foundation of possible worlds theory is that reality is a universe consisting of multiple worlds. At the center there is the actual world and orbiting around it are possible worlds, which are the sum of everything imaginable, but not actually existing. In this same way a fictional text can be considered a universe, which consists of a textual actual world in the center with textual alternative possible worlds circling around it. The textual actual world is what really happens in the text. Textual alternative possible worlds on the other hand can be counterfactuals, dreams or embedded narratives (Ryan 2006, 644-647; Ryan 1991, 23-25; Koskimaa 2000, 66-67).

This, however, is based on realist fiction. What makes time loops different is that possible worlds can become actual. The agent in a time loop does not only imagine different possible outcomes to a situation but in fact experiences them (again, either directly or indirectly). Each time the agent enters a possible world, it turns into actuality. The agent then actualizes several possible worlds, one at a time. After she exits the actualized world, it returns to the state of possibility and another world is actualized. In this way past paradoxically becomes a state of possibility even though it also actually happened. If looking it from the perspective of the agent it is the actualized past, but if looking at it from the perspective of the fictional characters inhabiting the current actualized world, it is merely a possibility. In addition, since there is always only one physical manifestation of the agent in each moment in time, the travel between the worlds must happen in mind, not body. This idea is based on Ryan (2006, 663) and Koskimaa $(2000,71)$ who postulate that a way for a fictional character to move from one parallel world to another is to merge her consciousness with her counterpart in another world.

We can illustrate the basic structure of a time loop narrative with figure 2. It is a simplified figure of the structure of a time loop story where the agent is inside the story. In this figure the agent goes through three possible worlds. Because time loops often have a time limit, let us imagine, for example, that the agent can spend a maximum of eight minutes in each world. So she repeats a time period of eight minutes maximum three times, always in another possible world. 
The zigzagging arrow represents the agent's movement through the worlds. For her the future is always where the arrow's head points and the past is where the arrow's tail lies. The dotted lines between the worlds represent the traveling between the worlds. The lines are dotted because this traveling is typically not narrated. The agent merely appears in the next world. The agent starts at minute 0 in the first possible world. She spends there the maximum time of 8 minutes, but fails at what she is trying to accomplish. Then she actualizes the possible world number 2 , starting from the beginning. Here she dies before 8 minutes has passed, so she moves to the third world. The arrow continues past 8 minutes in the third possible world. This is because that is the world the agent stays in, so the world stays actualized for her. The looping stops in this world and time continues past eight minutes. (Fig. 2)

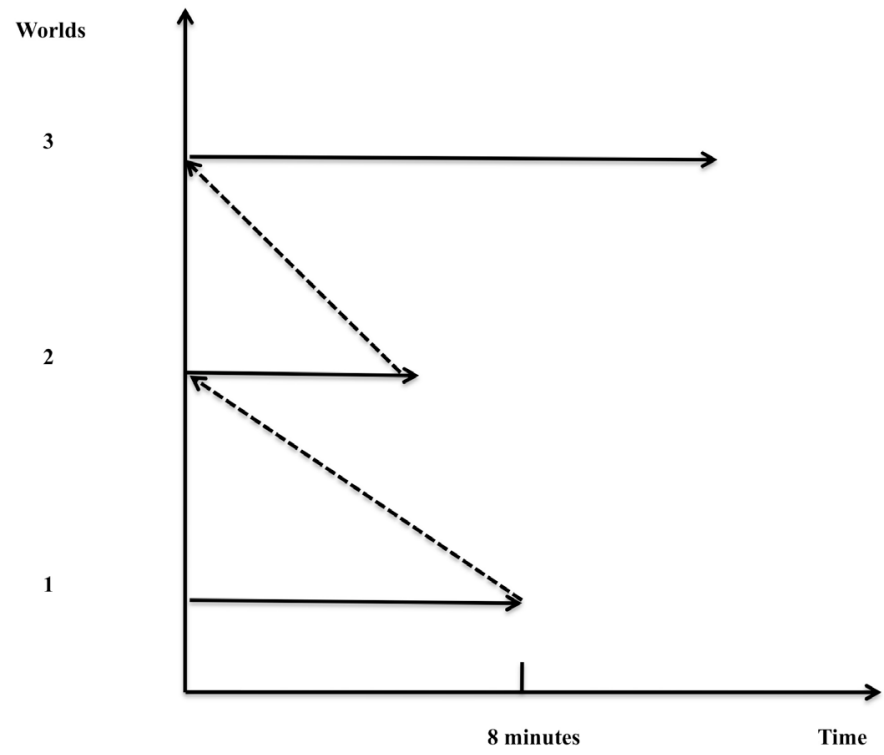

Figure 2. Agent inside a time loop story

As stated before, it is also possible for only an outside agent to experience the loop. Schenk $(2013,34)$ juxtaposes Groundhog Day with Super Mario 
Bros. That is, the player, much like the protagonist of the film, can return to an earlier point in time and make use of the skills or knowledge she gained from the previous game session. I will now proceed to conceptualize the time loop in the case of Super Mario Bros and other linear video games with retry mechanics. The figure 3 illustrates this option. Each actualization of the game session occurs in a possible world and the boxes represent each of these actualizations. The boxes are separate because they are not part of the same narrative, just different actualizations of the game. The player, who is the agent, is outside the diegesis of the game but her perspective is aligned with Mario's different counterparts in different possible worlds. Mario himself is not aware of the repetition (if we assume that inside the fictional world Mario is a conscious person), since he only experiences everything once. Federico Igarzábal Alvarez $(2016,232)$ calls this discrepancy of information between the player and avatar as temporal paradox: the future for the avatar is the past for the player. (Fig. 3)

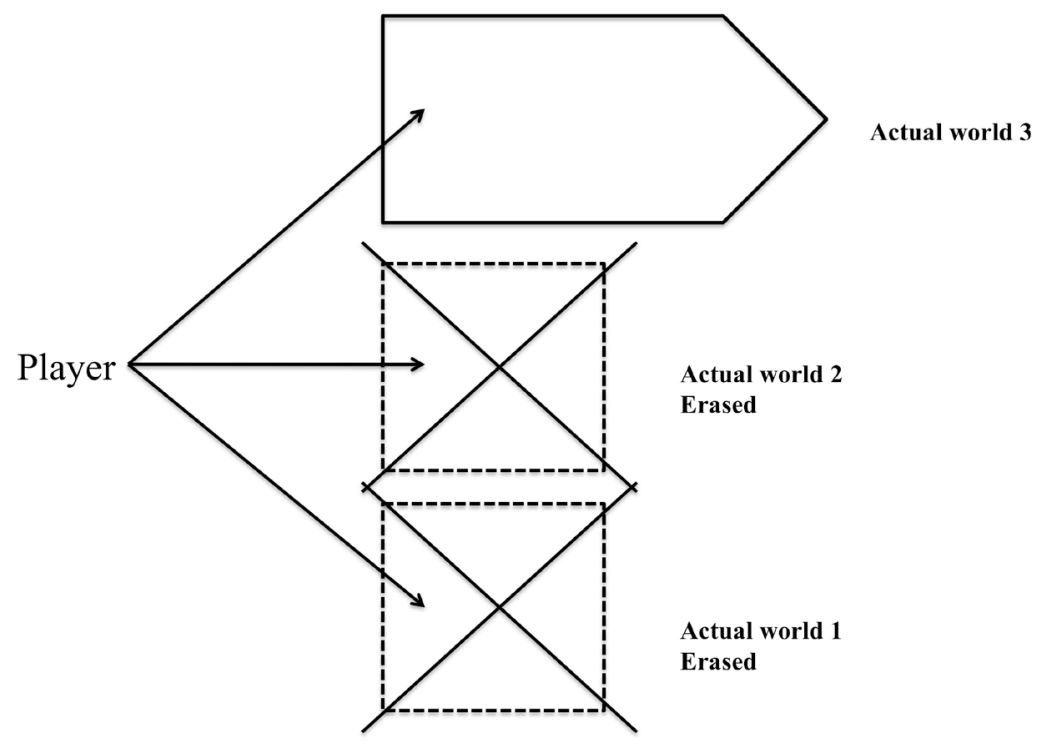

Figure 3. Agent outside a linear narrative 
In the first game session the player actualizes a possibility where Mario bumps into a mushroom and dies. When the player repeats the level, she actualizes a second possible turn of events. This time she uses the knowledge from previous actualization and avoids the mushroom. However she miscalculates and Mario falls into a pit and dies. Third time turns out to be the charm: the player has learnt from her mistakes and passes the level. The last box is in the shape of an arrow because this is from where the game continues and what stays actualized in the narrative. The failed tries before it are erased.

The player has now actualized three possible outcomes of the same events, and has repeated a specific period of time. She has thus experienced a time loop. However, the two failed actualizations are not part of the narrative of the game, only the game session. They are actualized but erased. The narrative in all its simplicity is that Mario will rescue the princess. It is not that Mario will either rescue the princess or die. This does not change the fact that the player really actualized worlds inside the game where Mario died.

According to Jesper Juul (1998) the ending of a video game is often well known in advance and the player's goal is to actualize that ending. This corresponds well with time loop narratives which are usually linear narratives, with no multiple endings. The looping character needs to learn something and use the knowledge and skills to actualize the best of possible worlds. The repetition is merely what enables her to actualize the ending. For example in the time loop film Edge of Tomorrow it is clear from early on that the goal of the protagonist is to win the war against aliens and save the world. The enjoyment of viewing the film is then not guessing the outcome but seeing how the protagonist arrives there, similarly to Super Mario Bros games.

However, a multilinear narrative can also appear as a time loop for an extradiegetic agent. This mostly applies to video games but also to Choose Your Own Adventure books and hypertexts. Now if we turn to look at figure 4 , it consists of two images of the same branching structure. The agent is above, outside of the story, again controlling an avatar's counterparts in possible worlds. The first time the player completes the game (the image on the left), her actions and choices actualize a set of possible worlds. The actualized path is on the left side of the branching structure with bold lines. The set of worlds not actualized remains a possibility and is shown as dotted lines. (Fig. 4) 

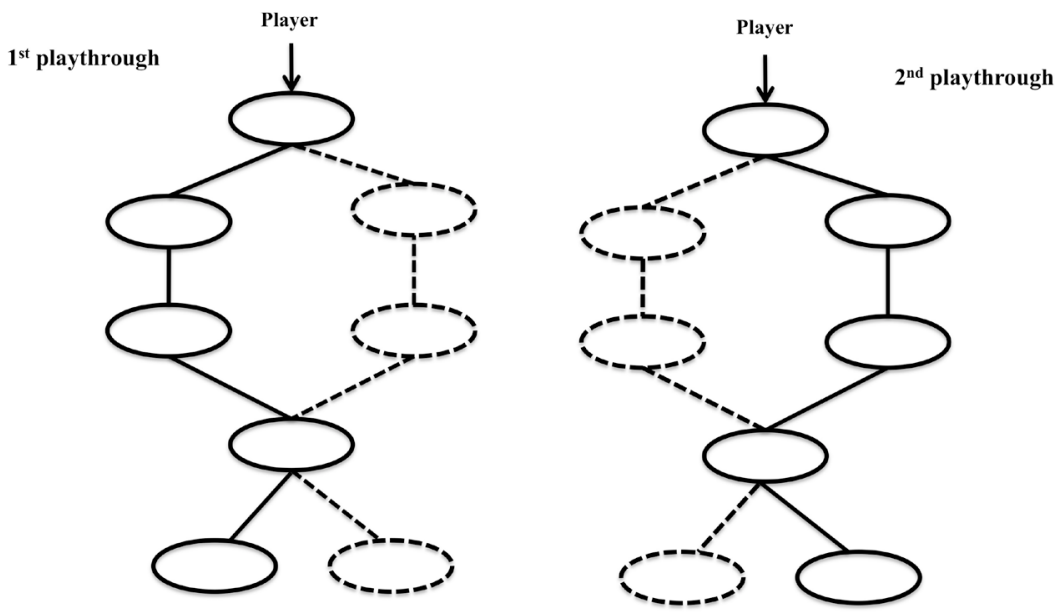

Figure 4. Agent outside a multilinear narrative

This is not yet a time loop, but it becomes one if the player plays the game again. Now on the second time around (the image on the right), the player makes different choices based on her previous experiences and she has a set of skills and knowledge acquired from the first playthrough. This learning by repetition is a defining characteristic of a time loop. The player then actualizes the set of possible worlds on the right. The left path of possible worlds is now only a possibility. Unlike with linear video games the actualization of possible worlds occurs inside the story, but the looping is still experienced outside the story.

It is interesting to note that the narrative in these games follows the forking paths structure. That is, the protagonist (or protagonists) splits into various versions of herself every time the story forks. It is the addition of the extradiegetic agent who experiences the repetition, which turns this into a time loop. The repetition in the branching structure is probably less concrete than in linear narratives, since different choices lead to different paths. However, in practice the different branches often overlap so the player will need to repeat some of the same scenes as well. To further complicate this structure, many video games with branching structure also have retry mechanics. 
$1^{\text {st }}$ playthrough

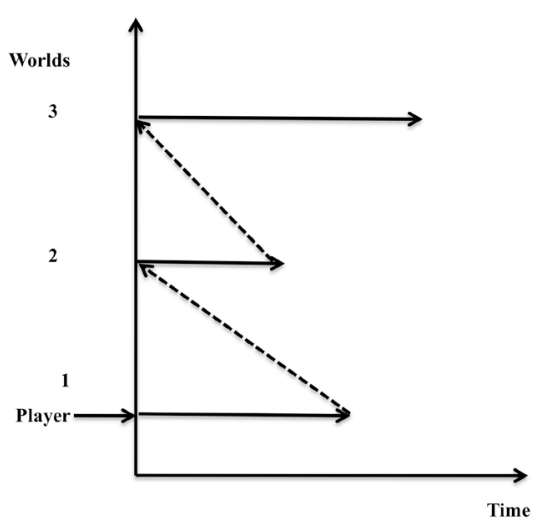

$2^{\text {nd }}$ playthrough

Fictional universe 2

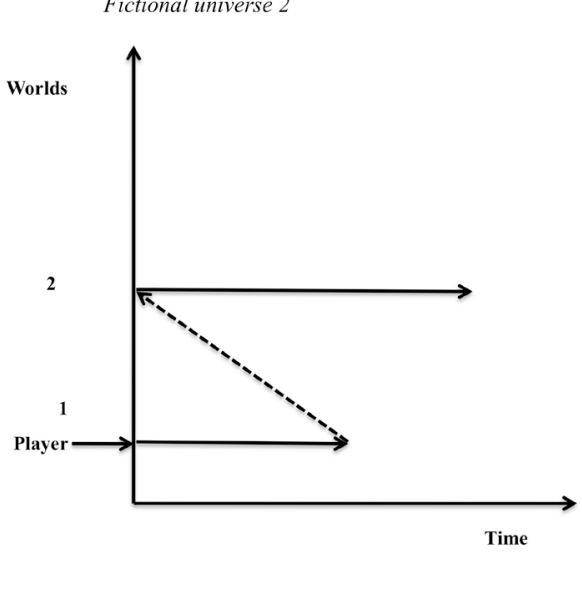

Figure 5. Agent inside and outside of a narrative

The third and final example is a situation where there is an agent both outside and inside the diegesis. This is a more rare case than options one and two. An example of a video game with a time loop narrative is Life Is Strange. It is a story, told in 5 episodes, about a girl named Max who has the power to manipulate time. Her most important ability in the game is that she can rewind time. The player then spends much of the game rewinding and replaying scenes. Since Max is aware of the looping, it is Max who merges with her counterparts in possible worlds while being controlled by the player. Figure 5 illustrates this situation. Since Life Is Strange is a time loop narrative, the figure resembles previously shown figure 2 . The difference is that there is now a player outside of the diegesis controlling the avatar. In addition, the time limit for repetition is missing since each case of replay can be of individual length. This is not unique to Life Is Strange but also applies to other time loop narratives in video games, such as Prince of Persia: Sands of Time (Ubisoft Montreal 2003). (Fig. 5)

However, the structure of the game is much more complex than this figure would show. The story and gameplay consist of numerous repetitions throughout the narrative, the actual number of repetitions would depend 
on each individual player. Instead of then representing the entirety of the narrative, like figure 2 does in cases of less interactive narratives like films, this figure only represents one instance of replay. In this example (the figure on the left) the player (and Max) need three tries to succeed in fulfilling a goal and then the story continues. The story of Life Is Strange consists of several of these repetitions with varying lengths. The story is further complicated by the fact that the entirety of the narrative can either form a time loop or not, depending on the choices of the player. The story then has forks in addition to loops, as well as other kind of time manipulation. Life Is Strange brings out the fact that time loop does not need to expand the entirety of the narrative, it can also only be one of many tropes used. This seems to be especially true with video games, although also some time travel narratives in other media include time loops as only part of the story.

There are two additional observations to be made of Life Is Strange from the point of view of fictional ontology. Figure 5 shows the same replay incident twice. This is to illustrate that despite the fact that the player and the avatar are aligned and aware of the looping, the player as the outside agent is higher in hierarchy. If the player replays the game or parts of it, then each time that happens, she actualizes a new possible fictional universe. These replays are not anymore part of the same story, but separate actualizations of it. Each time the game is replayed the avatar Max splits into her counterpart located in a parallel fictional universe and these counterparts are not aware of each other. Max is only aware of the looping that happens inside the fictional universe she is situated in ${ }^{4}$. The player then experiences a multiplied loop if replaying the game and is always more knowledgeable than the avatar Max. This is why the scene in the figure is only repeated once in the second gameplay session.

The other interesting observation is that Max (and the player) has the ability to merge possible worlds. Max can rewind time around her so that it does not affect her current location. For example in one scene she (and the player) blows up a door in order to enter a locked room. After entering the room she must now rewind time back to the point where the door was still intact. Even though she accomplishes this, she herself stays inside the room. In this case the effect is like having two possible worlds melting into one: the world where the door never was blown up and the world where Max

${ }^{4}$ This situation to some extent resembles how various parts of transmedia productions may or may not 'remember' events which have taken place in other parts (Harvey 2015, 2).

International Journal of Transmedia Literacy - 4 - December 2018 http: //www.ledonline.it/transmedialiteracy - Online ISSN 2465-2261 - Print ISSN 2465-227X 
entered the room by blowing up the door. In similar fashion, Max can acquire an object and then rewind back to the time she had not yet acquired the object but now the object is with her. By this merging of worlds Max and the player literally get the benefit of best of both worlds. Similar merger can be found from other time loop narratives in video games too, such as Braid (Number None 2008) but it is not common for time loop narratives in other media.

\section{THE PARADOX OF MUTUALLY EXCLUSIVE EVENTS}

"I've killed myself so many times I don't even exist anymore", states Phil Connors (Bill Murray) in Groundhog Day. In the course of one repeated day he jumps off a building, is hit by a car, electrocutes himself in the bath and drives off a cliff taking an innocent groundhog with him. Regardless of that every morning he wakes up without a scratch; everything he did in the previous loop is erased. The paradox however is that Phil Connors did experience everything and it all stays in his memory. He then has memories of events that happened in other realities. Koskimaa (2000, 66-73), in his study of hypertexts, calls this leaking of ontological borders ontolepsis. It can be experienced by a character inside the fictional universe, but also a reader of a hypertext, or in this case, a player. In this section I will give an example on how ontolepsis affects the agent inside and outside of the narrative.

In Life Is Strange the protagonist Max is repeatedly faced with the death of her best friend Chloe. For example, in one possible world Chloe is left paralyzed after a car accident. Chloe asks Max to help her die and she reluctantly obliges. Max then travels back in time to prevent the accident thus actualizing a possible world where Chloe is alive and well. Max is portrayed as happy to see Chloe alive but also haunted by the knowledge that in another world she helped Chloe die. This burden of living through all the possibilities, alone, is a recurring theme in the game narrative. Near the end of the story Max is lost in a nightmare where all her experiences and decisions from other worlds come to torment her.

Since Life Is Strange is a video game there is an additional level of ontolepsis for the player to experience. In episode 2 there is a scene where Kate, a friend of Max, tries to commit suicide by jumping off a building. Depending on what player makes Max do or say, not just in the scene but 
also throughout the episode, Kate either lives or dies. If Max fails to convince Kate to live, she will jump off the building and die. Max and the player cannot rewind time and undo the jump. So in the fictional universe of the game, the world where Kate kills herself will stay actualized ${ }^{5}$. However, since the player is higher in ontological hierarchy, she can, if she wishes, play the whole episode again, making different choices to actualize a universe where Kate lives. But player is now alone with the experience of Kate both dying and living because for Max the situation can only go one way in her fictional universe.

The statistics at the end of the episode reveal that $65 \%$ of players saved Kate but it does not reveal how many of them did it on the first try. In any case that means $35 \%$ of players did not go back and save her. According to a blog text and the comments it received (see Klepek 2016), the reasoning behind some of the players not going back and saving Kate was that it did not feel honest. One, of course, should not make too wide generalizations based on one blog text, but it presents an interesting idea that the first experience can feel more authentic and valuable than the replay of it. The reaction of these players emphasizes the differences between the ontological positions of the agent outside the diegesis and the agent inside it. For the fictional character the events in the storyworld are of course real. The player, on the other hand, knows she is playing a game so authenticity becomes valuable, even if it costs a life of a character. So in these story games it is not only the exploration of different possibilities that matters to the extradiegetic agent, it is also the emotional experience and the possibility to experience a coherent narrative.

\section{CONCLUSIONS}

In this article I have discussed time loop as a phenomenon both fictional characters in time loop narratives and players of video games can experience. My objective has been to juxtapose the time loop in video games and films, as well as to analyze the practical implications of mutually exclusive events for the player of a video game. My first aim was to conceptualize the time loop from the point of view of the character and player. My analysis

${ }^{5}$ In later episodes of the game, Kate's suicide however can be undone but it comes with a price.

International Journal of Transmedia Literacy - 4 - December 2018

http: //www.ledonline.it/transmedialiteracy - Online ISSN 2465-2261 - Print ISSN 2465-227X 
revealed that there are three alternatives to experiencing the loop. It can be experienced only by a fictional character in a time loop narrative, or only by a person who plays a video game with replay mechanics or it can be experienced by character and player both. These different ontological levels demonstrate how the looping can occur just on the level of the story, just on the level of the mechanics or both.

The practical insight gained from the analysis is the fact that point of view matters when discussing narrative structures. Forking paths and time loops are both game mechanics and future narratives. They resemble each other but differ in focalization. However, this grows complicated in the context of video games because of players' unique position. According to Juul (2001) players inhabit a twilight zone where they undertake a role inside the game while existing outside of it. Forking paths structure of a video game can then appear as a time loop from the point of view of players if they replay the game. This is because players can use what they learnt from earlier playthroughs to implement change. Vice versa, a video game with a time loop narrative can be a forking paths narrative for the character, if replayed. That is, the character is only aware of the looping inside the playthrough but she is not aware whether the game is played again. If the player replays the game, it is not the same character but her counterpart in another fictional universe that functions as the avatar.

The second aim of my article was to use the time loop to illustrate how the mechanics and conventions from video games can get remediated and incorporated into the story of films and other non-playable media. Most notable connection between a video game and a time loop film is in the structure where the character, like the player of a linear video game, needs to actualize the correct ending. The events that do not belong to the narrative (eg. avatar death) first happen and are then erased. The influence of video games, however, can also be observed in other aspects. An important similarity is the way time loop narratives approach death. Instead of portraying the death of the protagonist as tragic and final, it is presented unsentimentally, often even in a comic light, resembling the way player characters die in video games. This is because, like in video games, death is just a temporary glitch on the way to victory. Sometimes the protagonist, like the player character, can even choose to die just so she can go back and do things differently. Time loop narratives also borrow from video games the way they portray the supporting characters. Like the player of a video game, also the looping protagonist, is the only one aware of the repetition 
and thus the only one having agency. The other characters are like nonplayer characters in video games; they keep repeating the same things unless the protagonist/player character intervenes.

The imitation and inspiration, though, flow both ways. Life is Strange is influenced by a forking paths film The Butterfly Effect (2004) as well as Groundhog Day. The game is peppered with intermedial references to these films, and Life is Strange even pays homage to Groundhog Day by mentioning it by name (see Life is Strange References). In wider context the influence of films can be seen in the ambitious scripts of some narrative driven video games, where the composition is closer to an interactive movie than a game. It may also be argued, that transmedia productions which include both games and fixed narratives, are creating media use habits where expectations from one media form are easily expanded to other forms. An example of this being game-like looping which is easily recognized in contemporary cinema. The overlap between transmedia elements (same story passages told e.g. in cinema, novel, comic) also creates kinds of time loops, as the user experiences the same stories several times, told through different media. It is important to state though that stories with repetition and mutually exclusive events have existed long before video games were even invented. Films such as Edge of Tomorrow and Run Lola Run clearly remediate video games in their aesthetics and level-like structures, whereas in films like Groundhog Day the resemblance seems to be more incidental. It can be postulated that playfulness and learning from mistakes, as well as what if scenarios are typical to the human condition and games and films offer different ways to express and explore that.

The third aim of my article was to analyze the paradoxical experience of something both happening and not happening and its effects on the player and character. This quality can make the story of a film more playful and game-like but also tragic, if the character has to experience the repeated loss of a loved one, and existential as it shakes the foundations of reality and our responsibility in shaping our own lives. The difference between forking paths narratives in films and video games is that the film director can decide which of the possible futures bears the most weight. This is accomplished by showing the most important version last. That is because, according to David Bordwell (2002, 100-103), story logic dictates that what comes last in the story, is the ending. The last fork always bears the most weight because it is by default the ending. The audience trusts that the director has placed the alternative events in certain order for a reason, but 
in the case of video games, the player lacks that authority. She can actualize the different branches of the story in random order and all versions of the events can feel equally valid, therefore lacking the emotional impact of a proper ending.

In these video games the retry mechanics can become a hindrance to enjoying the story; leaving the narrative crippled by its media format. If there are several ways for the story to play out and the player explores more than one of them, the narrative might lose its emotional impact and lead to the feeling of inauthenticity. Game designers are aware of this dilemma. David Cage, for example, the lead designer and screenwriter of a forking paths video game Heavy Rain (Quantic Dream, 2010), has wished people would play it only once. He states (2009): "Life you can only play once. When you make choices, you rarely have a chance to go back and say 'Hey, what if I was doing something else?' Well, you know what? You made the choice; that's it. I would like people to have this experience".

There are, however, games which cherish the possibility of repetition and replay, by making the alternative events an integral part of the playing experience. Life is Strange accomplishes this by first of all incorporating the retry mechanics into the diegesis so that the protagonist is aware of the looping. This, though, is not yet where Life is Strange stands out, since games such as Prince of Persia and Braid also have a protagonist aware of the looping. But unlike them, Life is Strange is not a linear game with one correct path to follow, it is instead a forking paths narrative. The protagonist of Life is Strange is then aware of both the loops and the forks. This makes the player and the game character more aligned than in many other games (although still not equal) and the revisiting of choices becomes part of the gameplay.

Instead of taking us to a world, like our own, where you cannot go back and alter your choices, Life is Strange shows us how it might be if you could do just that. The game underlines that there often are no right or wrong choices but all choices have both wanted and unwanted consequences. Furthermore, the protagonist of the game keeps on questioning her choices and as the story progresses she is increasingly haunted by what happened in other possible worlds. The video game format of Life is Strange, then, does not diminish the power of its story but on the contrary, enhances it, since both player and protagonist need to face the ambiguity of a world where choices can be undone and there is no authority to determine what path is the right one. 


\section{REFERENCES}

Alvarez Igarzábal, Federico. 2016. "The Groundhog Day Effect: Iterations in Virtual Space”. In Time to Play: Zeit im Computerspiel, edited by Stefan Höltgen and Jan Claas van Treeck, 225-246. Glückstadt: Verlag Werner Hülsbusch.

Bal, Mieke. 1997. Narratology: Introduction to the Theory of Narrative. Toronto: Univ. of Toronto Press.

Before I Fall. 2017. Directed by Ry-Russo Young. USA: Awesomeness Films and Jon Shestack Productions.

Bode, Christoph and Rainer Dietrich. 2013. Future Narratives, Volume 1: Theory, Poetics, and Media-Historical Moment. Berlin/Boston: De Gruyter.

Bolter, J. David and Richard A. Grusin. (1999) 2000. Remediation: Understanding new media. Cambridge: Mit Press.

Bordwell, David. 2002. "Film Futures”. SubStance 31(1): 88-104.

Cage, David and Guillaume de Fondamiere. 2009. "Changing the Game: The Quantic Dream Heavy Rain Interview Part Two”. By Billy Berghammer, G4TV. http://g4tv.com/games/ps3/36147/heavy-rain/articles/68230/Changing-The-Game-The-Quantic-Dream-Heavy-Rain-Interview-Part-Two. Accessed 14 Jun. 2016.

Chatman, Seymour. 1978. Story and Discourse: Narrative Structure in Fiction and Film. Ithaca: Cornell Univ. Press.

Life is Strange. 2015. Dontnod Entertainment.

Edge of Tomorrow. 2014. Directed by Doug Liman. USA: Warner Bros, et al., 2014.

Genette, Gérard. 1980. Narrative Discourse. Translated by Jane E. Lewin. Ithaca: Cornell Univ. Press.

Groundhog Day. 1993. Directed by Harold Ramis. USA: Columbia Pictures.

Harvey, Colin B. 2015. Fantastic Transmedia. Palgrave Macmillan UK.

Jenkins, Henry. 2007. "Transmedia Storytelling 101”. Confessions of an ACA-Fan. http://henryjenkins.org/blog/2007/03/transmedia_storytelling_101.html. Accessed 12 Nov 2017.

Juul, Jesper. 1998. "A Clash Between Game and Narrative”. Jesperjuul.net. http://www.jesperjuul.net/text/clash_between_game_and_narrative.html. Accessed 15 Jun. 2016.

Juul, Jesper. 2001. "Games telling stories? A brief note on games and narratives". Game studies 1.1. http://www.gamestudies.org/0101/juul-gts/ Accessed 14 Nov. 2017.

Kallay, Jasmina. 2013. Gaming Film: How Games Are Reshaping Contemporary Cinema. Basingstoke: Palgrave Macmillan. 
Klastrup, Lisbeth, and Susana Tosca. 2014. "Game of Thrones: Transmedial worlds, fandom, and social gaming". In Storyworlds across Media, edited by Marie-Laure Ryan and Jan-Noël Thon, 295-314. Nebraska: University of Nebraska Press.

Klepek, Patrick. 2015. "Life Is Strange Can Go Way, Way Wrong”. Kotaku. http://kotaku.com/life-is-strange-can-go-way-way-wrong-1696574304. Accessed 15 Jun. 2016.

Koskimaa, Raine. 2000. Digital literature: From Text to Hypertext and Beyond. Jyväskylä: University of Jyväskylä.

Life is Strange References. http://life-is-strange.wikia.com/wiki/References. Accessed 14 of Oct. 2017.

Lola Rennt. 1998. Directed by Tom Tykwer. Germany: X-Filme Creative Pool, et al.

Navarro-Remesal, Victor and Shaila García-Catalán. 2015. "Try Again: The Time Loop as a Problem-Solving Process in Save the Date and Source Code”. In Time Travel in Popular Media: Essays on Film, Television, Literature and Video Games, edited by Matthew Jones and Joan Ormrod, 209-222. Jefferson, NC: Mcfarland \& Company.

Super Mario Bros. 1985. Nintendo.

Braid. 2008. Number None.

Oliver, Lauren. 2010. Before I Fall. London: Hodder \& Stoughton.

Parshall, Peter. 2012. Altman and After: Multiple Narratives in Film. Lanham, MD: Scarecrow Press.

Przypadek. 1981. Directed by Krzysztof Kieslowski. Poland: P.P. Film Polski.

Heavy Rain, 2010. Quantic Dream.

Rajewsky, Irina. 2005. "Intermediality, intertextuality, and remediation: A literary perspective on intermediality". Intermédialités: histoire et théorie des arts, des lettres et des techniques/Intermediality: History and Theory of the Arts, Literature and Technologies 6: 43-64.

Ryan, Marie-Laure. 2006. From Parallel Universes to Possible Worlds: Ontological Pluralism in Physics, Narratology and Narrative. Poetics Today 27(4), 633674.

Ryan, Marie-Laure. 1991. "Possible Worlds, Artificial Intelligence, and Narrative Theory". Bloomington and Indianapolis: Indiana Univ. Press.

Schenk, Sabine. 2013. Future Narratives, Volume 3: Running and Clicking. Future Narratives in Film. Berlin/Boston, DE: De Gruyter.

Sliding Doors. 1998. Directed by Peter Howitt. UK and USA: Intermedia Films et al.

Source Code. 2011. Directed by Duncan Jones. USA: Vendome Pictures, et al.. 
Tocci, Jason. 2008. "You are dead. Continue? Conflicts and Complements in Game Rules and Fiction”. Eludamos. Journal for Computer Game Culture 2(2), 187201.

The Butterfly Effect. 2004. Directed and written by Eric Bress and J. Mackye Gruber. USA: FilmEngine, et al..

Prince of Persia: Sands of Time. 2003. Ubisoft Montreal.

Xena The Warrior Princess. 1995-2001. Directed by Garth Maxwell, et al. USA: MCA Television. 